\title{
1 Hippocampal Subfields in Adolescent Anorexia Nervosa
}

2

3 *Anna D. Myrvang ${ }^{\mathrm{a}, \mathrm{g}}$ Torgil R. Vangberg ${ }^{\mathrm{a}, \mathrm{b}}$, Kristin Stedal ${ }^{\mathrm{c}}, \varnothing_{\text {yvind R }} \varnothing^{\mathrm{c}, \mathrm{f}}$, Tor Endestad ${ }^{\mathrm{d}}$, Jan

4 H. Rosenvinge ${ }^{\mathrm{a}}$, Per M. Aslaksen ${ }^{\mathrm{a}, \mathrm{e}}$.

5

7 Norway, Troms $\varnothing$, Norway.

$8 \quad \mathrm{~b}$ Department of Clinical Medicine, University Hospital of North Norway, Norway.

$9 \quad{ }^{\mathrm{c}}$ Regional Department for Eating Disorders, Division of Mental Health and Addiction, Oslo

10 University Hospital, Norway.

$11{ }^{\mathrm{d}}$ Department of Psychology, Faculty of Social Sciences, University of Oslo, Norway.

$12{ }^{\mathrm{e}}$ Regional Center for Eating Disorders, University hospital of North Norway, Norway.

13 Institute of Clinical Medicine, University of Oslo, Norway

$14{ }^{\mathrm{g}}$ Athinoula A. Martinos Center for Biomedical Imaging, Massachusetts General Hospital,

15 Boston, USA.

16

17 * Corresponding author

18 Anna Dahl Myrvang

19 Address: UiT The Arctic University of Norway, Huginbakken 32, N-9037, Norway

20 Telephone number: +4777625232

21 E-mail: anna.d.myrvang@uit.no 
Abstract

23 Patients with anorexia nervosa (AN) exhibit volume reduction in cerebral gray matter (GM),

24 and several studies report reduced hippocampus volume. The hippocampal subfields (HS) are

25 functionally and structurally distinct, and appear to respond differently to neuropathology.

26 The aim of this study was to investigate HS volumes in adolescent females with restrictive

27 AN compared to a healthy age-matched control group (HC). The FreeSurfer v6.0 package was

28 used to extract brain volumes, and segment HS in 58 female adolescents ( $\mathrm{AN}=30, \mathrm{HC}=28)$.

29 We investigated group differences in GM, white matter (WM), whole hippocampus and 12

30 HS volumes. AN patients had significantly lower total GM and total hippocampal volume. No

31 group difference was found in WM. Volume reduction was found in 11 of the $12 \mathrm{HS}$, and

32 most results remained significant when adjusting for global brain volume reduction.

33 Investigations of clinical covariates revealed statistically significant relationships between the

34 whole hippocampus, several HS and scores on depression and anxiety scales in AN. Results

35 from this study show that young AN patients exhibit reduced volume in most subfields of the

36 hippocampus, and that this reduction may be more extensive than the observed global cerebral 37 volume loss.

38

39 Keywords:

40 Anorexia Nervosa; MRI; Hippocampus; FreeSurfer; Brain segmentation. 
42 Anorexia nervosa (AN) is a severe mental health disorder characterized by a disturbance in body image perception and a restriction of nutrient intake resulting in abnormally low body weight (American Psychiatric Association, 2013). Patients with AN have significantly elevated mortality rates compared to other mental health disorders (Arcelus et al., 2011) and the majority have their illness debut during adolescence. Brain imaging studies consistently find that global gray matter (GM) volume is reduced in patients with AN, although there are some discrepancies regarding the degree of atrophy and affected areas (Gaudio et al., 2011; King et al., 2015; Seitz et al., 2016). A recent meta-analysis concluded that GM reduction is significantly greater in adolescent patients with AN compared to adults (Seitz et al., 2016). Findings regarding white matter (WM) are inconsistent, but recent studies suggest that WM volume and integrity are better preserved in young patients with AN compared to adults (Pfuhl et al., 2016; Seitz et al., 2016). Longitudinal studies indicate that total brain volume mostly normalizes as patients recover (Bernardoni et al., 2016; Mainz et al., 2012), but it is yet unclear whether regeneration is total and if it applies to all cerebral regions.

Volume reduction of the hippocampus formation has been reported in several studies in both adults (Burkert et al., 2015; Chui et al., 2008; Connan et al., 2006; King et al., 2015; Mainz et al., 2012). The formation of the hippocampus is well known for its involvement in learning and memory, but also plays an important role in emotional regulation (Fanselow and Dong, 2010). Hippocampal atrophy is evident in other severe mental health disorders, such as major depression (Treadway et al., 2015), schizophrenia (Wright et al., 2000), bipolar disorder (Haukvik et al., 2015), post-traumatic stress disorder (PTSD) (Hayes et al., 2017) and borderline personality disorder (Driessen et al., 2000) and a common underlying mechanism driven by stress and elevated glucocorticoid levels has been proposed (Sapolsky, 2000). Patients with AN often experience comorbid symptoms of depression and anxiety (Kaye et al., 2004; O'Brien and Vincent, 2003). The link between hippocampal volume reduction and comorbid symptoms has not been extensively investigated. One study found no relationship between depression and coping and hippocampus volume in adult AN (Burkert et al., 2015).

The hippocampus is a heterogeneous structure with multiple cell layers and several distinct "hippocampal subfields" (HS) that are structurally and functionally different from one another (Duncan et al., 2012; Leutgeb et al., 2007; Zeineh et al., 2000; Zhu et al., 2017). Advanced new methods for segmentation of the hippocampus enable examination of the HS separately. The FreeSurfer v6.0 hippocampal subfields atlas was built from ultra-high 
can model 13 segments, and has been shown to perform well in neurodegenerative disease populations (Iglesias et al., 2015).

A number of neuroimaging studies have investigated HS separately in disease populations and found that neuropathology can affect these regions differently. Among patients with severe mental health disorders, the most frequently reported findings are volume reduction in the CA structures, the subiculum and dentate gyri (Haukvik et al., 2015; Hayes et al., 2017; Ho et al., 2017; Ota et al., 2017; Treadway et al., 2015). A recent study found that Cornu Ammonis 1 (CA1) volume was reduced in early stages of schizophrenia, but that atrophy spread to other subfields as the illness progressed (Ho et al., 2017), indicating that duration of illness may be an important factor to consider when studying volume reduction in the hippocampus in mental health disorders.

To our knowledge, only one previous study has investigated HS in AN patients (Burkert et al., 2015). Adult AN patients who had been ill for several years were found to have a significant reduction in the fimbria - a white matter bundle projecting along the anterior-posterior axis of the hippocampus (Burkert et al., 2015), and an increase in the size of the hippocampal fissure - the "ventricle" of the hippocampus. Recent studies suggest that variability in duration of $\mathrm{AN}$, which typically debuts in adolescents, may lead to different findings in neuroimaging studies of adults and adolescent (Pfuhl et al., 2016; Seitz et al., 2016). It is therefore of interest to investigate the hippocampus and HS volumes in the early stages of AN.

The studies that have reported hippocampal atrophy in AN (Burkert et al., 2015; Connan et al., 2006; Giordano et al., 2001; Mainz et al., 2012) vary in their methods of correction for individual differences in brain volume. None of the reported studies have aimed to investigate the selective effect of AN on the hippocampus by adjusting for the observed global brain volume reduction. It remains unclear whether the hippocampus is particularly affected in $\mathrm{AN}$, or if the volume reduction in the hippocampus is a consequence of the observed global volume reduction. Furthermore, methods of segmentation vary and results from the manual delineation of HS can be particularly difficult to replicate (Van Leemput et al., 2009). Further investigation is needed to reveal the relationship between AN and the hippocampus and its subfields.

The aim of the current study was to examine HS in young patients in an early stage of AN. We investigated 12 subfields segmented by the hippocampal subfields segmentation tool in the FreeSurfer software package (Iglesias et al., 2015) - a fully automated algorithm. We expected to find that adolescent AN patients had volume reduction in total cerebral GM and 
109 the whole hippocampus compared to healthy age-matched controls. We expected to find a

110 selective HS volume reduction and an increased fissure, similar to what has been found

111 previously in adult AN patients (Burkert et al., 2015). Furthermore, we investigated if HS

112 volumes were significantly smaller in AN patients when adjusting for total brain volume -

113 which we expected to be reduced in AN. As HS volume reduction is also found in mental

114 health disorders that often occur as comorbid conditions in AN patients, we wished to further

115 explore the association between HS volume, AN symptoms and symptoms of anxiety and

116 depression. We expected to find a negative relative relationship between HS volumes and 117 symptoms of depression and anxiety.

118 


\section{$120 \quad$ 2.1. Study design and sample}

121 Inpatients with AN were recruited from the Regional Center for Eating Disorders at the

122 University Hospital of North Norway (RSS) and Oslo University Hospital (RASP). In total,

12333 female patients with $\mathrm{AN}$ (Age: $\mathrm{M}=15.8, \mathrm{SD}=1.7$ ) and 30 female healthy age-matched

124 controls (Age: $\mathrm{M}=16.2, \mathrm{SD}=1.9$ ) were recruited for the study (10 patients and 10 controls

125 were tested and scanned at RASP). Healthy controls (HC) were recruited from local high

126 schools. Neuropsychological testing and scanning was conducted less than two weeks apart.

127 All participants were scanned in the evening between $3 \mathrm{pm}$ and $8 \mathrm{pm}$.

128 Inclusion criteria for AN patients were the DSM-V criteria for restrictive AN (no

129 history of binge-purge episodes), diagnosis set by a clinical specialist in psychology or

130 medicine. Age-adjusted, standardized body mass indexvalues (BMI-SDS) were calculated

131 using Norwegian normative data from the Bergen Growth Study (Júlíusson et al., 2013). A

132 measure of body mass index increase between admission and scanning (BMI-increase) was

133 calculated by subtracting body mass index (BMI) at admission from BMI at the day of

134 scanning. Exclusion criteria for all participants were neurological disorders and organic brain

135 injury, history of bulimia nervosa, schizophrenia, psychotic episodes and the use of

136 antipsychotic medication. Additional exclusion criteria for $\mathrm{HC}$ were lifetime or current eating

137 disorders or obesity (BMI > 30).

\subsection{Ethics}

140 The Norwegian Committee for Medical and Health Research Ethics (REC), North region

141 approved the study, under protocol number 302969. Informed, written consent was obtained

142 from all participants. Parents also gave written consent for participants < 16 years of age.

\subsection{Image acquisition}

145 MR scanning was performed with a 3T Siemens Magnetom Skyra Syngo MR D13C at the

146 University Hospital of Tromsø and with a Phillips Achieva 3T scanner at the University

147 Hospital of Oslo. At both sites, high resolution 3D T1-wheighted images were acquired. In

148 Troms $\varnothing$, we used a magnetization-prepared rapid gradient-echo (MPRAGE) sequence with

149 the following parameters: Orientation = Sagittal; No. of slices =176; Voxel size = 1 × 1 × 1 ;

150 Slice thickness $=1 \mathrm{~mm}$; repetition time $(\mathrm{TR})=2300 \mathrm{~ms}$; echo time $(\mathrm{TE})=2.98 \mathrm{~ms}$; field of

151 view $(\mathrm{FOV})=256 \times 256$; Flip angle $=9^{\circ}$; and inversion time $(\mathrm{TI})=900 \mathrm{~ms}$. In Oslo, a 3D

152 sequence was used for acquisition with the following parameters: Orientation = Sagittal; No 
of slices $=184 ;$ Voxel size $=1 \times 1 \times 1$; Slice thickness $=1 \mathrm{~mm} ; \mathrm{TR}=2300 \mathrm{~ms} ; \mathrm{TE}=2.98 \mathrm{~ms}$; $\mathrm{FOV}=256 \times 256$; Flip angle $=8^{\circ}$; and TI $=900 \mathrm{~ms}$.

\subsection{Image processing}

Surface reconstruction and volumetric segmentation was performed with FreeSurfer v6.0 software (http://surfer.nmr.mgh.harvard.edu) version 6.0; Fischl et al. 2002, Fischl et al., 2004) with the recon-all processing pipeline and the hippocampal subfields module (Iglesias et al., 2015). The pipeline includes motion correction, normalization to Talairach space, intensity bias correction, skull-stripping, surface registration and segmentation. Two of the authors (TRV and ADM) visually inspected image registration results.

\subsubsection{Selected brain volumes}

The following 12 HS are modeled by the FreeSurfer hippocampal subfields atlas (Iglesias et al., 2015) and were investigated in this study: The CA1, CA2/3, CA4, the molecular layer of the CA regions (ML), the Granule Cell layer of the Dentate Gyrus (GCDG), the pre-, parasubiculum, and the subiculum, the hippocampus-amygdala transition area (HATA), the fimbria, the hippocampal fissure and the hippocampal tail (Figure 1). We also investigated total GM and WM volumes, estimated total intracranial volume (eTIV) and whole brain volume (ventricles excluded).

\subsection{Mental health}

The Norwegian versions of the Beck's Depression Inventory (BDI-II) (Beck et al., 1988), and the State-Trait Anxiety Inventory (STAI) forms Y1 (state anxiety) and Y2 (trait anxiety) (Spielberger et al., 1970) was used to measure symptoms of depression and anxiety, respectively. The Eating Disorder Examination Questionnaire (EDE-Q) (Fairburn and Beglin, 2008) was used to measure eating disorder symptoms. The EDE-Q consists of four subscales (restriction, concerns about eating, weight and figure) and a global scale. The MiniInternational Neuropsychiatric interview (M.I.N.I) 6.0 (Sheehan et al., 1998) was used to screen for comorbid mental health disorders before patients were assessed by a clinical specialist in psychology or medicine. IQ was measured by Wechslers Adult Intelligence Scale IV (WAIS-IV) or Wechslers Intelligence Scale for Children IV (WISC-IV) for participants $<16$ years of age (Wechsler, 2008, 2003).

\subsection{Statistical analyses}


187 We performed tests of normality and inspected plots for all variables and found no violations of the assumptions for parametric tests. Group differences in demographic variables and psychometric measures were investigated by one-way analysis of variance. Linear regression analyses were used to investigate group differences on global GM and WM, adjusted for age, drug use and scanner site. Inspections of the cortical surface and subcortical volumes revealed a substantial spread of cortical volume reduction and volume reduction in several subcortical structures. To investigate whether brain volumes were affected by scanner site, we performed linear regression analyses using only $\mathrm{HC}$ participants with total GM and the whole hippocampus, adjusted for age and eTIV, as the outcome variables and scanner site as the independent variable. Scanner site, adjusted for age and eTIV, was not associated with total GM ( $b=0.02, p=0.44)$ or left hippocampus $(b=-0.27, p=0.21)$, but was close to significant in the right hippocampus $(b=-0.39, p=0.05)$. We adjusted for site in all further analyses. As an additional measure against the potential confounding effect of site, we re-performed the main analyses of hippocampus and HS in a subsample with participants from one scanner only (Supplement tables 1-2).

A series of linear regression analyses was performed to investigate group differences in the whole hippocampus and HS volumes, averaged across hemispheres. All analyses were also performed separately for the two hemispheres. To adjust for potential confounding effect of age dispersion, depressive symptoms, individual differences in intracranial volume, psychopharmacological treatment and the two different scanners, the variables age, BDI-II score, eTIV, drug use and scanner site were entered as covariates. In a secondary series of analyses, we replaced eTIV with whole brain volume as a covariate to investigate whether volume reduction in the whole hippocampus and HS was affected by total brain volume. All analyses were also repeated with STAI-Y1 (measuring state anxiety symptoms) score replacing the depression score to adjust for potential confounding effect of anxiety symptoms.

213 we conducted group stratified linear regression analyses of all HS volumes that were 214 significantly smaller in the AN group and the following variables: BMI, BMI-SDS, BMI215 increase, Weeks since admission (to inpatient care), Years since first GP consultation 216 (regarding eating disorder symptoms), EDE-Q (four subscales and global scale) BDI-II, STAI 217 Y1. In all models we added age, scanner site, drug use and eTIV as covariates to adjust for 218 potential confounding effects. All results were corrected for errors of multiple comparisons 219 with the false discovery rate (FDR) method using a syntax for SPSS (http://www- 
01.ibm.com/support/docview.wss?uid=swg21476447) and a false discovery rate with $\mathrm{q}=0.05$. All statistical analyses were performed using IBM SPSS 24.

\section{Results}

The AN group had significantly higher scores on self-report measures of mental illness and significantly lower BMI and BMI-SDS (Table 1). Linear regression analysis of global GM and WM volumes showed that AN patients had significantly reduced volume in cerebral GM and total brain volume. No group differences were found in cerebral WM and eTIV (Table 2).

All HS volumes except for the hippocampal fissure were significantly explained by group affiliation adjusted for site, age, depression score (BDI-II), drug use and eTIV, and remained significant after FDR correction (Tables 3-4). In the secondary analysis, where eTIV was replaced by brain volume as a covariate, the fimbria and the hippocampal tail where no longer significantly explained by group affiliations after correction for multiple comparisons (Table 4). When adjusting for anxiety, results were similar for the eTIV adjusted analyses, but none of the HS remained significant when adjusting for total brain volume (Supplement table 3). We conducted the same analyses on a subgroup collected from one single scanner $(\mathrm{N}=41)$ to avoid the potential confound of scanner variability and results showed similar results for the eTIV adjusted analyses, but none of the HS were significantly explained by group affiliation when adjusting for whole brain volume (Supplement table 1-2). Because we did not have a hypothesis about lateralization of volume reduction and because the results for the two hemispheres were highly similar, only results from analyses performed on volumes averaged across hemispheres are presented.

In the group stratified regression analyses of HS of interest and clinical measures (BMI, BMI increase, duration of inpatient care, AN symptom duration, scores from EDE-Q, BDI-II and STAI measuring AN symptoms, depression and anxiety) results revealed significant relationships between BDI, STAI Y1 and several HS (Table 5). No significant associations were found regarding BMI and EDE-Q scores (Table 5), or any of the other ANrelated measures. We did not find any statistically significant associations between HS volumes and clinical measures in the HC group.

\section{Discussion}

The aim of the present study was to investigate hippocampal subfields in adolescents with restrictive AN compared to healthy age-matched controls. We found statistically significant

253 volume reductions in all but one of the investigated HS volumes when adjusting for age, 
depression score (BDI-II), scanner site and eTIV. Results showed that the AN group had smaller CA areas and less volume in the presubiculum, the molecular layers of the CA areas, the HATA and the GCDG. Most results remained significant also when adjusting for global brain volume which was expectedly reduced in the AN sample. This might indicate that the volume reduction in the hippocampus is more extensive than the general brain volume reduction, and that this structure is particularly vulnerable in AN. The fissure was not increased in the AN group as found in a previous study of adult AN patients (Burkert et al., 2015). In their study of adult patients, Burkert et al. found volume reduction only in the fimbria and our results seem to indicate that hippocampus reduction is more extensive in adolescent AN patients and not specific to selected subfields. The reason for the discrepancy might be the young age of our sample and that the developing brain may respond differently to illness debut. Another explanation could be that GM areas normalize after the initial acute phase of AN. Our results are consistent with findings regarding global GM in AN. A recent meta-analyses of volumetric studies in AN found that adolescents had significantly greater GM volume loss compared to adults (Seitz et al., 2016).

The use of different hippocampal segmentation methods complicates the comparison of the results of studies of HS. In their study of adult AN patients, Burkert and colleagues (Burkert et al., 2015) used FreeSurfer version 5.3, which performs a more crude segmentation and does not model all of the subfields. The previous version has been criticized for not agreeing well with volumes from histological studies (Schoene-Bake et al., 2014). The FreeSurfer v6.0 atlas is an improvement to previous atlases in that it is made from higher resolution images and is built from more cases, makes no assumptions about acquisition parameters and can model more subfields than any other atlas (Iglesias et al., 2015).

Stress and excessive glucocorticoid exposure is often reported in severe mental health disorders and is proposed as the driving mechanism of hippocampal atrophy (Mondelli et al., 2010; Sapolsky, 2000; Videbech and Ravnkilde, 2004; Watanabe et al., 2017). Higher selfreported stress levels have been found to be associated with greater hippocampus reduction in major depressive patients (Treadway et al., 2015), and higher serum cortisol levels were

282 found in first-episode depressive patients (Watanabe et al., 2017). Excessive hormone production can lead to volume reduction in the hippocampus, as seen in patients with the hypercorticolism disease Cushing's syndrome (Starkman et al., 1992). Patients with AN often have comorbid depression and anxiety disorders (Kaye et al., 2004), report higher stress levels (Burkert et al., 2015) and have elevated cortisol levels (Mainz et al., 2012) and it is possible that this is also driving volume reduction in AN. In the present study, the potential confound 
of depression was addressed by adjusting for BDI-II score in the main analyses of HS. The group effect was still present with this adjustment, indicating that depressive symptoms in our sample is not driving volume reduction in the hippocampus. Similar results were found when adding anxiety scores as a covariate, but none of the results from analyses with adjustments for whole brain volume remained significant after correction for multiple comparisons. These results may have been significant in a larger sample.

Group stratified analyses revealed significant, positive relationships between several HS and symptoms of depression and anxiety measured by BDI II and STAI Y1, and Y2, showing that patients with larger HS volumes had higher scores for these measures, indicating more severe symptoms. No such relationships were found in the HC group. These findings were somewhat unexpected since previous studies have found a reduction in hippocampus volume to be associated with depression and PTSD (Hayes et al., 2017; Treadway et al., 2015). However, the relationship between depression and HS volume appear to be a matter of duration and not severity - i.e. more depressive episodes is associated with greater volume loss (Treadway et al., 2015). Depression in AN is found to be highly related to core symptoms of the disorder such as body dissatisfaction, and the assessment of comorbidity between these disorders is challenging (Espelage et al., 2003). Very few patients in our sample received a comorbid diagnosis according to the M.I.N.I interview, in spite of high scores on BDI and STAI. Furthermore, it is possible that patients that experienced less symptoms of depression and anxiety prior to admission will experience more emotional distress from being admitted to inpatient care. The patients in our study were recently admitted and scores on depression and anxiety scales may have been temporarily elevated due to the new imposed weight rehabilitation regimen. The relationship between symptoms of depression and anxiety and HS in our sample may thus be driven by related factors such as stress and coping mechanisms.

The contribution of low BMI and emaciation to hippocampal volume loss in AN is unclear. Findings regarding global GM volume are inconsistent, but some studies have identified significant correlations with BMI (Seitz et al., 2015), lowest lifetime BMI and degree of weight loss prior to admission (Bomba et al., 2013). In addition, the fact that brain volume tends to normalize when body weight is restored (King et al., 2015; Mainz et al., 2012) suggests that weight is a contributing factor in global cerebral volume reduction. One study found regional volume reductions in the ACC but not global GM (Mühlau et al., 2007) suggesting that some regions may be more vulnerable to malnourishment. In line with the previous study on HS (Burkert et al., 2015), we did not find a significant relationship between BMI and hippocampal volume. 
A limitation to our study is the use of two different scanners - a probable confounder of the results. To account for this, we re-performed the main analyses on a subgroup from only one scanner. These results were similar to the results from the main analyses, indicating that scanner site did not affect the main outcome in a large extent. However, the subgroup analyses had a low $\mathrm{N}(\mathrm{AN} \mathrm{N}=21)$ and this may not be sufficient to detect group differences. Although the most recent version of the FreeSurfer HS atlas used in this study is an improvement upon the previous version, there still are limitations regarding the boundaries between some of the subfields, for example the CA-fields. The CA4 and the dentate gyrus also overlap in the atlas, and it might not be possible to distinguish these two subfields practically. The atlas was built from manual delineations in elderly subjects and might not perform as well in younger populations (Iglesias et al., 2015).

Further limitations of our study were that we did not have data available to control for variations in pretest severity of illness, notably periods of marked weight loss (i.e. a BMI < 17) and lowest lifetime BMI or comorbidity prior to admission. The patients in our study had been admitted for a mean duration of 4.5 weeks with a large dispersion ( $\mathrm{SD}=4.0$ weeks) and were likely to have been on weight rehabilitation programs for several weeks. The mean BMI of 16.3 ( $\mathrm{SD}=1.6)$ in the AN group suggests that not all of the patients were in the most acute phase of their illness. However, we did not find a significant association between BMI increase score, measured by subtracting the BMI at admission from the BMI at the day of the scan, and the HS, indicating that hippocampus volumes were not affected by patients' weight gain during the first weeks of inpatient treatment.

The present study is the first to investigate hippocampal subfields selectively in adolescent AN patients in an early stage of illness. The most important finding was that several HS were found to be significantly reduced in adolescent patients with AN compared to healthy controls. The effect was present when adjusting for depression and anxiety, suggesting that the extensive HS volume reduction in AN that is not driven by depression or anxiety. However, no AN characteristic variables were associated with the observed volume reduction. The positive association between depression and anxiety might be a result of associated factors such as stress and coping mechanisms. Future studies should include more elaborate measures of comorbidity and AN symptomatology, particularly measures of stress and coping. 


\section{Declaration of interest}

355 All authors declare no conflicts of interest.

356

\section{Acknowledgements}

358 We would like to thank all participants and contributors from the Regional Center for Eating

359 Disorders at the University Hospital of North Norway and Oslo University Hospital.

360

\section{Funding}

362 This project is funded by the Research Council of Norway, P.O. Box 564, NO-1327 Lysaker,

363 Norway. Program: KVINNEHELSE. Project number: 229142.

364

365 
American Psychiatric Association, 2013. Diagnostic and Statistical Manual of Mental Disorders, Arlington. https://doi.org/10.1176/appi.books.9780890425596.744053

Arcelus, J., Mitchell, A.J., Wales, J., Nielsen, S., 2011. Mortality rates in patients with anorexia nervosa and other eating disorders. A meta-analysis of 36 studies. Arch. Gen. Psychiatry 68, 724-31. https://doi.org/10.1001/archgenpsychiatry.2011.74

Beck, A.T., Steer, R.A., Carbin, M.G., 1988. Psychometric properties of the Beck Depression Inventory: Twenty-five years of evaluation. Clin. Psychol. Rev. 8, 77-100. https://doi.org/10.1016/0272-7358(88)90050-5

Bernardoni, F., King, J.A., Geisler, D., Stein, E., Jaite, C., Nätsch, D., Tam, F.I., Boehm, I., Seidel, M., Roessner, V., Ehrlich, S., 2016. Weight restoration therapy rapidly reverses cortical thinning in anorexia nervosa: A longitudinal study. Neuroimage 130, 214-222. https://doi.org/10.1016/J.NEUROIMAGE.2016.02.003

Bomba, M., Riva, A., Veggo, F., Grimaldi, M., Morzenti, S., Neri, F., Nacinovich, R., 2013. Impact of speed and magnitude of weight loss on the development of brain trophic changes in adolescents with anorexia nervosa: a case control study. Ital. J. Pediatr. 39, 14. https://doi.org/10.1186/1824-7288-39-14

Burkert, N.T., Koschutnig, K., Ebner, F., Freidl, W., 2015. Structural hippocampal alterations, perceived stress, and coping deficiencies in patients with anorexia nervosa. Int. J. Eat. Disord. 48, 670-676. https://doi.org/10.1002/eat.22397

Chui, H.T., Christensen, B.K., Zipursky, R.B., Richards, B.A., Hanratty, M.K., Kabani, N.J., Mikulis, D.J., Katzman, D.K., 2008. Cognitive Function and Brain Structure in Females With a History of Adolescent-Onset Anorexia Nervosa. Pediatrics 122, e426-e437. https://doi.org/10.1542/peds.2008-0170

Connan, F., Murphy, F., Connor, S.E.J., Rich, P., Murphy, T., Bara-Carill, N., Landau, S., Krljes, S., Ng, V., Williams, S., Morris, R.G., Campbell, I.C., Treasure, J., 2006. Hippocampal volume and cognitive function in anorexia nervosa. Psychiatry Res. Neuroimaging 146, 117-125. https://doi.org/10.1016/j.pscychresns.2005.10.006 Driessen, M., Herrmann, J., Stahl, K., Zwaan, M., Meier, S., Hill, A., Osterheider, M., Petersen, D., 2000. Magnetic Resonance Imaging Volumes of the Hippocampus and the Amygdala in Women With Borderline Personality Disorder and Early Traumatization. Arch. Gen. Psychiatry 57, 1115. https://doi.org/10.1001/archpsyc.57.12.1115

Duncan, K., Ketz, N., Inati, S.J., Davachi, L., 2012. Evidence for area CA1 as a match/mismatch detector: A high-resolution fMRI study of the human hippocampus. 
Hippocampus 22, 389-398. https://doi.org/10.1002/hipo.20933

Espelage, D.L., Mazzeo, S.E., Aggen, S.H., Quittner, A.L., Sherman, R., Thompson, R., 2003. Examining the construct validity of the Eating Disorder Inventory. Psychol. Assess. 15, 71-80. https://doi.org/10.1037/1040-3590.15.1.71

Fairburn, C.G., Beglin, S., 2008. Eating Disorder Examination Questionnaire (EDE-Q 6.0), in: Fairburn, C.G. (Ed.), Cognitive Behavior Therapy and Eating Disorders. Guilford Press, New York, pp. 309-313. https://doi.org/10.1016/j.eatbeh.2009.09.005

Fanselow, M.S., Dong, H.W., 2010. Are the Dorsal and Ventral Hippocampus Functionally Distinct Structures? Neuron 65, 7-19. https://doi.org/10.1016/j.neuron.2009.11.031 Fischl, B., Salat, D.H., Busa, E., Albert, M., Dieterich, M., Haselgrove, C., van der Kouwe, A., Killiany, R., Kennedy, D., Klaveness, S., Montillo, A., Makris, N., Rosen, B., Dale, A.M., 2002. Whole brain segmentation: automated labeling of neuroanatomical structures in the human brain. Neuron 33, 341-55.

Fischl, B., Van Der Kouwe, A., Destrieux, C., Halgren, E., Ségonne, F., Salat, D.H., Busa, E., Seidman, L.J., Goldstein, J., Kennedy, D., Caviness, V., Makris, N., Rosen, B., Dale, A.M., 2004. Automatically Parcellating the Human Cerebral Cortex. Cereb. Cortex 14, 11-22. https://doi.org/10.1093/cercor/bhg087

Gaudio, S., Nocchi, F., Franchin, T., Genovese, E., Cannatà, V., Longo, D., Fariello, G., 2011. Gray matter decrease distribution in the early stages of Anorexia Nervosa restrictive type in adolescents. Psychiatry Res. Neuroimaging 191, 24-30. https://doi.org/10.1016/j.pscychresns.2010.06.007

Giordano, G.D., Renzetti, P., Parodi, R.C., Foppiani, L., Zandrino, F., Giordano, G., Sardanelli, F., 2001. Volume measurement with magnetic resonance imaging of hippocampus-amygdala formation in patients with anorexia nervosa. J. Endocrinol. Invest. 24, 510-514. https://doi.org/10.1007/BF03343884

Haukvik, U.K., Westlye, L.T., Mørch-Johnsen, L., Jørgensen, K.N., Lange, E.H., Dale, A.M., Melle, I., Andreassen, O.A., Agartz, I., 2015. In vivo hippocampal subfield volumes in schizophrenia and bipolar disorder. Biol. Psychiatry 77, 581-588. https://doi.org/10.1016/j.biopsych.2014.06.020

Hayes, J.P., Hayes, S., Miller, D.R., Lafleche, G., Logue, M.W., Verfaellie, M., 2017. Automated measurement of hippocampal subfields in PTSD: Evidence for smaller dentate gyrus volume. J. Psychiatr. Res. 95, 247-252. https://doi.org/10.1016/J.JPSYCHIRES.2017.09.007

Ho, N.F., Iglesias, J.E., Sum, M.Y., Kuswanto, C.N., Sitoh, Y.Y., De Souza, J., Hong, Z., 
Fischl, B., Roffman, J.L., Zhou, J., Sim, K., Holt, D.J., 2017. Progression from selective to general involvement of hippocampal subfields in schizophrenia. Mol. Psychiatry 22, 142-152. https://doi.org/10.1038/mp.2016.4

Iglesias, J.E., Augustinack, J.C., Nguyen, K., Player, C.M., Player, A., Wright, M., Roy, N., Frosch, M.P., McKee, A.C., Wald, L.L., Fischl, B., Van Leemput, K., 2015. A computational atlas of the hippocampal formation using ex vivo, ultra-high resolution MRI: Application to adaptive segmentation of in vivo MRI. Neuroimage 115, 117-137. https://doi.org/10.1016/j.neuroimage.2015.04.042

Júlíusson, P.B., Roelants, M., Nordal, E., Furevik, L., Eide, G.E., Moster, D., Hauspie, R., Bjerknes, R., 2013. Growth references for 0-19 year-old Norwegian children for length/height, weight, body mass index and head circumference. Ann. Hum. Biol. 40, 220-227. https://doi.org/10.3109/03014460.2012.759276

Kaye, W.H., Bulik, C.M., Thornton, L., Barbarich, N., Masters, K., 2004. Comorbidity of anxiety disorders with anorexia and bulimia nervosa. Am. J. Psychiatry 161, 2215-21. https://doi.org/10.1176/appi.ajp.161.12.2215

King, J.A., Geisler, D., Ritschel, F., Boehm, I., Seidel, M., Roschinski, B., Soltwedel, L., Zwipp, J., Pfuhl, G., Marxen, M., Roessner, V., Ehrlich, S., 2015. Global cortical thinning in acute anorexia nervosa normalizes following long-term weight restoration. Biol. Psychiatry 77, 624-32. https://doi.org/10.1016/j.biopsych.2014.09.005

Leutgeb, J.K., Leutgeb, S., Moser, M.-B., Moser, E.I., 2007. Pattern Separation in the Dentate Gyrus and CA3 of the Hippocampus. Science (80-. ). 315, 961-966. https://doi.org/10.1126/science.1135801

Mainz, V., Schulte-Ruther, M., Fink, G.R., Herpertz-Dahlmann, B., Konrad, K., 2012. Structural Brain Abnormalities in Adolescent Anorexia Nervosa Before and After Weight Recovery and Associated Hormonal Changes. Psychosom. Med. 74, 574-582. https://doi.org/10.1097/PSY.0b013e31824ef10e

Mondelli, V., Pariante, C.M., Navari, S., Aas, M., D’Albenzio, A., Di Forti, M., Handley, R., Hepgul, N., Marques, T.R., Taylor, H., Papadopoulos, A.S., Aitchison, K.J., Murray, R.M., Dazzan, P., 2010. Higher cortisol levels are associated with smaller left hippocampal volume in first-episode psychosis. Schizophr. Res. 119, 75-78. https://doi.org/10.1016/j.schres.2009.12.021

Mühlau, M., Gaser, C., Ilg, R., Conrad, B., Leibl, C., Cebulla, M.H., Backmund, H., Gerlinghoff, M., Lommer, P., Schnebel, A., Wohlschläger, A.M., Zimmer, C., Nunnemann, S., 2007. Gray matter decrease of the anterior cingulate cortex in anorexia 
nervosa. Am. J. Psychiatry. https://doi.org/10.1176/appi.ajp.2007.06111861

O’Brien, K.M., Vincent, N.K., 2003. Psychiatric comorbidity in anorexia and bulimia nervosa: nature, prevalence, and causal relationships. Clin. Psychol. Rev. 23, 57-74. https://doi.org/10.1016/S0272-7358(02)00201-5

Ota, M., Sato, N., Hidese, S., Teraishi, T., Maikusa, N., Matsuda, H., Hattori, K., Kunugi, H., 2017. Structural differences in hippocampal subfields among schizophrenia patients, major depressive disorder patients, and healthy subjects. Psychiatry Res. Neuroimaging 259, 54-59. https://doi.org/10.1016/j.pscychresns.2016.11.002

Pfuhl, G., King, J.A., Geisler, D., Roschinski, B., Ritschel, F., Seidel, M., Bernardoni, F., Müller, D.K., White, T., Roessner, V., Ehrlich, S., 2016. Preserved white matter microstructure in young patients with anorexia nervosa? Hum. Brain Mapp. 37, 40694083. https://doi.org/10.1002/hbm.23296

Sapolsky, R.M., 2000. Glucocorticoids and Hippocampal Atrophy in Neuropsychiatric Disorders. Arch. Gen. Psychiatry 57, 925-935. https://doi.org/10.1001/archpsyc.57.10.925

Schoene-Bake, J.-C., Keller, S.S., Niehusmann, P., Volmering, E., Elger, C., Deppe, M., Weber, B., 2014. In vivo mapping of hippocampal subfields in mesial temporal lobe epilepsy: Relation to histopathology. Hum. Brain Mapp. 35, 4718-4728. https://doi.org/10.1002/hbm.22506

Seitz, J., Herpertz-Dahlmann, B., Konrad, K., 2016. Brain morphological changes in adolescent and adult patients with anorexia nervosa. J. Neural Transm. 123, 949-959. https://doi.org/10.1007/s00702-016-1567-9

Seitz, J., Walter, M., Mainz, V., Herpertz-Dahlmann, B., Konrad, K., von Polier, G., 2015. Brain volume reduction predicts weight development in adolescent patients with anorexia nervosa. J. Psychiatr. Res. 68, 228-37. https://doi.org/10.1016/j.jpsychires.2015.06.019

Sheehan, D. V, Lecrubier, Y., Sheehan, K.H., Amorim, P., Janavs, J., Weiller, E., Hergueta, T., Baker, R., Dunbar, G.C., 1998. The Mini-International Neuropsychiatric Interview (M.I.N.I.): the development and validation of a structured diagnostic psychiatric interview for DSM-IV and ICD-10. J. Clin. Psychiatry 59(20), 22-33. https://doi.org/10.1016/S0924-9338(99)80239-9

Spielberger, C.D., Gorusuch, R.L., Lushene, R.E., 1970. Manual for the State-Trait Anxiety Inventory. Consulting Psychologists Press, Palo Alto, CA.

Starkman, M.N., Gebarski, S.S., Berent, S., Schteingart, D.E., 1992. Hippocampal formation 

volume, memory dysfunction, and cortisol levels in patients with Cushing's syndrome. Biol. Psychiatry 32, 756-765. https://doi.org/10.1016/0006-3223(92)90079-F

Treadway, M.T., Waskom, M.L., Dillon, D.G., Holmes, A.J., Park, M.T.M., Chakravarty, M.M., Dutra, S.J., Polli, F.E., Iosifescu, D. V., Fava, M., Gabrieli, J.D.E., Pizzagalli, D.A., 2015. Illness progression, recent stress, and morphometry of hippocampal subfields and medial prefrontal cortex in major depression. Biol. Psychiatry 77, 285-294. https://doi.org/10.1016/j.biopsych.2014.06.018

Van Leemput, K., Bakkour, A., Benner, T., Wiggins, G., Wald, L.L., Augustinack, J., Dickerson, B.C., Golland, P., Fischl, B., 2009. Automated segmentation of hippocampal subfields from ultra-high resolution in vivo MRI. Hippocampus 19, 549-557. https://doi.org/10.1002/hipo.20615

Videbech, P., Ravnkilde, B., 2004. Hippocampal volume and depression: a meta-analysis of MRI studies. Am. J. Psychiatry 161, 1957-66. https://doi.org/10.1176/appi.ajp.161.11.1957

Watanabe, R., Kakeda, S., Watanabe, K., Liu, X., Katsuki, A., Umeno-Nakano, W., Hori, H., Abe, O., Yoshimura, R., Korogi, Y., 2017. Relationship between the hippocampal shape abnormality and serum cortisol levels in first-episode and drug-naïve major depressive disorder patients. Depress. Anxiety 34, 401-409. https://doi.org/10.1002/da.22604

Wechsler, D., 2008. Wechsler Adult Intelligence scale - Fourth edition (WAIS-IV). Pearson, San Antonio, TX.

Wechsler, D., 2003. Wechslers Intelligence scale for Children - Fourth edition (WISC-IV). Psychological Corporation, San Antonio, TX.

Wright, I.C., Rabe-Hesketh, S., Woodruff, P.W.R., David, A.S., Murray, R.M., Bullmore, E.T., 2000. Meta-analysis of regional brain volumes in schizophrenia. Am. J. Psychiatry 157, 16-25. https://doi.org/10.1176/ajp.157.1.16

Zeineh, M.M., Engel, S.A., Bookheimer, S.Y., 2000. Application of Cortical Unfolding Techniques to Functional MRI of the Human Hippocampal Region. Neuroimage 11, 668-683. https://doi.org/10.1006/NIMG.2000.0561

Zhu, B., Chen, C., Dang, X., Dong, Q., Lin, C., 2017. Hippocampal subfields' volumes are more relevant to fluid intelligence than verbal working memory. Intelligence 61, 169 175. https://doi.org/10.1016/j.intell.2017.02.003 
Supplement table 1 Clinical measures in AN and HC for single scanner subgroup

\begin{tabular}{|c|c|c|c|c|}
\hline & $\begin{array}{c}\text { AN } \\
\text { Mean (SD) }\end{array}$ & $\begin{array}{c}\text { HC } \\
\text { Mean (SD) }\end{array}$ & F-value & $p$ \\
\hline $\mathrm{N}$ & 21 & 20 & & \\
\hline Age & $15.2(1.6)$ & $15.7(1.9)$ & 1.5 & .225 \\
\hline BMI & $16.1(1.4)$ & $21.8(3.1)$ & 60.4 & $<.001$ \\
\hline BMI-SDS & $-2.4(1.3)$ & $0.3(1.0)$ & 57.5 & $<.001$ \\
\hline Drugs $(\mathrm{SSRI} / \mathrm{GH})^{\mathrm{a}}$ & $2 / 2$ & 0 & & \\
\hline Left hand dominant & 1 & 3 & & \\
\hline Weeks since admission & $5.3(7.0)$ & - & & \\
\hline Years since first GP consult. & $1.1(1.2)$ & - & & \\
\hline
\end{tabular}

Supplement table 2 Hippocampal subfield volumes for adolescent AN and HC from single scanner subgroup

Adjusted for eTIV Adjusted for total brain volume

\begin{tabular}{lcccccc} 
Brain volume & Beta & $\boldsymbol{p}$ & R-square & Beta & $\boldsymbol{p}$ & R-square \\
\hline $\begin{array}{l}\text { Whole } \\
\text { hippocampus }\end{array}$ & -.424 & $\mathbf{. 0 0 1}$ & .558 & -.211 & .117 & .586 \\
Tail & -.399 & $\mathbf{. 0 1 1}$ & .304 & -.321 & .072 & .283 \\
Subiculum & -.216 & $\mathbf{. 1 4 8}$ & .323 & -.004 & .981 & .360 \\
Presubiculum & -.293 & $\mathbf{. 0 6 3}$ & .262 & -.084 & .615 & .348 \\
Parasubiculum & -.249 & $\mathbf{. 1 2 5}$ & .203 & -.023 & .891 & .309 \\
Fissure & -.069 & .672 & .182 & -.001 & .996 & .101 \\
CA1 & -.415 & $\mathbf{. 0 0 1}$ & .539 & -.197 & .142 & .588 \\
CA2-3 & -.373 & $\mathbf{. 0 0 6}$ & .493 & -.254 & .101 & .455 \\
CA4 & -.300 & $\mathbf{. 0 3 1}$ & .431 & -.148 & .352 & .407 \\
ML & -.439 & $\mathbf{. 0 0 1}$ & .549 & -.229 & .088 & .590 \\
GCDG & -.324 & $\mathbf{. 0 1 8}$ & .454 & -.157 & .311 & .443 \\
HATA & -.313 & $\mathbf{. 0 1 9}$ & .491 & -.059 & .666 & .561 \\
Fimbria & -.212 & $\mathbf{. 2 0 6}$ & .142 & -.012 & .946 & .226 \\
\hline
\end{tabular}

Note: Statistics: Linear regression analyses with two different adjustments for brain size: eTIV (estimated total intracranial volume) and total brain volume without ventricles. In both sets of analyses covariates are group affiliation (group variable was coded $\mathrm{AN}=0$ and $\mathrm{HC}=1$ ), age, depression score (BDI-II), scanner site and drug use. Variables presented in bold are significant after FDR correction for multiple comparisons. $\mathrm{CA}=$ Cornu Ammonis. $\mathrm{GCDG}=$ Granule cell layer of the dentate gyrus. HATA = Hippocampus-amygdala transition area. 
Supplement table 3 Hippocampus volumes in adolescent AN vs. HC adjusted for state anxiety (STAI-Y1)

\begin{tabular}{|c|c|c|c|c|c|c|}
\hline \multirow{2}{*}{ Brain volume } & \multicolumn{3}{|c|}{ Adjusted for eTIV } & \multicolumn{3}{|c|}{ Adjusted for total brain volume } \\
\hline & Beta & $p$ & R-square & Beta & $p$ & R-square \\
\hline \multicolumn{7}{|l|}{ Whole } \\
\hline hippocampus & -.599 & $<.001$ & .431 & -.371 & .014 & .538 \\
\hline Tail & -.413 & .016 & .303 & -.249 & .150 & .371 \\
\hline Subiculum & -.437 & .009 & .349 & -.266 & .122 & .378 \\
\hline Presubiculum & -.402 & .015 & .355 & -.232 & .164 & .414 \\
\hline Parasubiculum & -.378 & .026 & .312 & -.159 & .341 & .403 \\
\hline Fissure & -.035 & .840 & .232 & -.032 & .872 & .154 \\
\hline CA1 & -.544 & .001 & .417 & -.322 & .037 & .510 \\
\hline CA2-3 & -.523 & .003 & .316 & -.386 & .030 & .357 \\
\hline CA4 & -.505 & .004 & .285 & -.314 & .070 & .377 \\
\hline ML & -.627 & $<.001$ & .424 & -.411 & .008 & .526 \\
\hline GCDG & -.547 & .001 & .349 & -.343 & .037 & .444 \\
\hline HATA & -.538 & .001 & .413 & -.319 & .040 & .506 \\
\hline Fimbria & -.298 & .101 & .192 & -.157 & .413 & .209 \\
\hline
\end{tabular}

Note: Table shows results from linear regression analyses with two different adjustments for brain size: eTIV (estimated total intracranial volume) and total brain volume without ventricles. In both sets of analyses covariates are group affiliation (group variable was coded $\mathrm{AN}=0$ and $\mathrm{HC}=1$ ), age, state anxiety (STAI-Y1), scanner site and drug use. Variables presented in bold are significant after FDR correction for multiple commparisons. $\mathrm{CA}=$ Cornu Ammonis. $\mathrm{GCDG}=$ Granule cell layer of the dentate gyrus. HATA = Hippocampus-amygdala transition area. 
Table 1 Clinical measures in adolescent $\mathrm{AN}$ and $\mathrm{HC}$

\begin{tabular}{lcccc}
\hline Clinical measures & $\begin{array}{c}\text { AN } \\
\text { Mean }(\text { SD })\end{array}$ & $\begin{array}{c}\text { HC } \\
\text { Mean }(\text { SD })\end{array}$ & F-value & $\boldsymbol{p}$ \\
\hline $\mathrm{N}$ & 30 & 28 & & \\
Age & $15.8(1.7)$ & $16.2(1.9)$ & 0.9 & .343 \\
BMI & $16.3(1.6)$ & $21.8(3.1)$ & 73.9 & $<.001$ \\
BMI admission & $15.2(1.4)$ & - & & \\
BMI-increase & $0.9(0.6)$ & - & & \\
BMI-SDS & $-2.4(1.2)$ & $0.3(1.1)$ & 73.2 & $<.001$ \\
Drugs (SSRI/GH) & 7 & 0 & & \\
Left hand dominant & 2 & 2 & & \\
Weeks since admission* & $4.5(4.0)$ & - & & \\
Years since first GP & $1.6(1.4)$ & - & & \\
consult.** & & & & \\
& $101.1(12.0)$ & $104.0(8.2)$ & 292.0 & .068 \\
FSIQ* & $22.8(11.8)$ & $4.3(5.1)$ & 56.7 & $<.001$ \\
BDI II*** & $49.8(14.1)$ & $30.8(9.7)$ & 32.9 & $<.001$ \\
STAI Y1*** & $52.0(15.2)$ & $33.9(10.9)$ & 27.1 & $<.001$ \\
STAI Y2*** & $3.0(2.0)$ & $0.4(0.5)$ & 44.2 & $<.001$ \\
EDE-Q restriction** & $2.3(1.7)$ & $0.2(0.5)$ & 37.1 & $<.001$ \\
EDE-Q eating** & $3.0(1.8)$ & $0.7(0.8)$ & 36.3 & $<.001$ \\
EDE-Q weight** & $3.9(1.9)$ & $0.9(1.2)$ & 50.1 & $<.001$ \\
EDE-Q figure** & $3.0(1.7)$ & $0.6(0.6)$ & 53.7 & $<.001$ \\
EDE-Q global** & $1.0(1.2)$ & $0.1(0.3)$ & 17.8 & $<.001$ \\
Mini sum* & & & & \\
\hline
\end{tabular}

Note: Statistics: One-way ANOVA. BMI = Body mass index. BMI-SDS = Standardized BMI values based on Norwegian norms for children. ${ }^{\text {a }} 5$ subjects used Serotonin reuptake inhibitor (SSRI), 2 used growth hormones $(\mathrm{GH})$. Years since first GP consult $=$ Consultation concerning eating disorder symptoms. FSIQ $=$ Full Scale Intelligence Quotient. BDI = Becks Depression Inventory II. STAI $1 \& 2=$ State Trait Anxiety questionnaire form Y1 (State anxiety) and Y2 (Trait anxiety). EDE-Q = Eating Disorder Examination Questionnaire. MINI sum = Sum of diagnoses from MINI except Anorexia nervosa.*AN N $=29$. **AN N $=27$. ***AN N $=25$.

Table 2 Total brain volumes in adolescent AN and $\mathrm{HC}$

\begin{tabular}{lccccc}
\hline & $\begin{array}{c}\text { AN } \\
\text { Mean (SD) }\end{array}$ & $\begin{array}{c}\text { HC } \\
\text { Mean }(\text { SD) }\end{array}$ & Beta & $\boldsymbol{p}$ & $\begin{array}{c}\text { R- } \\
\text { square }\end{array}$ \\
Brain volumes & & & & & $\mathbf{7 7 6}$ \\
\hline Total gray matter & $\mathbf{6 6 2 8 1 2 . 9}(\mathbf{5 6 6 0 7 . 4})$ & $\mathbf{7 1 7 9 2 0 . 5}(\mathbf{5 9 5 8 6 . 9})$ & $\mathbf{- . 4 2 6}$ & $<.001$ & .681 \\
Cerebral white matter & $417027.1(47223.0)$ & $436765.0(46027.6)$ & -.100 & .246 & .681 \\
eTIV & $1452452.6(139298.6)$ & $1485015.9(121664.0)$ & -.118 & .360 & .142 \\
Total brain volume & $\mathbf{1 1 0 7 9 3 5 . 4 ( 9 1 5 4 0 . 3 )}$ & $\mathbf{1 1 8 4 7 3 5 . 0}(\mathbf{9 4 0 8 6 . 8})$ & $\mathbf{- . 4 0 9}$ & $\mathbf{. 0 0 1}$ & $\mathbf{. 2 4 7}$ \\
\hline
\end{tabular}

Note: Statistics: Linear regression adjusting for age, drug use and site. eTIV $=$ estimated total intracranial volume. Total gray and white matter was also adjusted for eTIV. Group variable was coded $\mathrm{AN}=0$ and $\mathrm{HC}=1$. Mean values are $\mathrm{mm}^{3} .{ }^{\mathrm{a}}$ Ventricles were excluded from total brain volume. 
Table 3 Hippocampus volumes in $\mathrm{mm}^{3}$ for adolescent $\mathrm{AN}$ and $\mathrm{HC}$

\begin{tabular}{lccc}
\hline Brain volumes & $\begin{array}{c}\text { AN } \\
\text { Mean }(\text { SD) }\end{array}$ & $\begin{array}{c}\text { HC } \\
\text { Mean (SD) }\end{array}$ & \% difference \\
\hline Whole hippocampus & $3327.7(299.8)$ & $3566.7(242.3)$ & $6.7 \%$ \\
$H S:$ & & & \\
Tail & $517.7(55.8)$ & $550.4(54.3)$ & $5.9 \%$ \\
Subiculum & $422.2(39.4)$ & $441.1(34.8)$ & $4.3 \%$ \\
Presubiculum & $307.4(29.7)$ & $326.7(26.6)$ & $5.9 \%$ \\
Parasubiculum & $62.8(8.1)$ & $68.1(6.3)$ & $7.8 \%$ \\
Fissure & $144.8(18.6)$ & $145.9(18.6)$ & $0.8 \%$ \\
CA1 & $610.3(69.4)$ & $661.7(60.2)$ & $7.8 \%$ \\
CA2-3 & $187.2(27.1)$ & $206.0(26.4)$ & $9.1 \%$ \\
CA4 & $241.0(26.2)$ & $258.0(22.7)$ & $6.6 \%$ \\
Molecular layer & $545.1(51.3)$ & $588.2(43.7)$ & $7.3 \%$ \\
GCDG & $280.7(29.9)$ & $301.4(25.5)$ & $6.9 \%$ \\
HATA & $61.2(8.9)$ & $67.1(6.5)$ & $8.8 \%$ \\
Fimbria & $92.0(12.7)$ & $98.1(13.2)$ & $6.2 \%$ \\
\hline
\end{tabular}

Note: Values are mean $\mathrm{mm}^{3}$ and standard deviations, averaged across hemispheres. $\mathrm{HS}=$ Hippocampal subfields. $\mathrm{CA}=$ Cornu Ammonis. $\mathrm{GCDG}=$ Granule cell layer of the dentate gyrus. HATA = Hippocampus-amygdala transition area. \% difference was calculated from mean volumes in $\mathrm{mm}^{3}(\mathrm{HC}-\mathrm{AN})$.

Table 4 Hippocampus volumes in adolescent AN vs. HC

\section{Adjusted for eTIV Adjusted for total brain volume}

\begin{tabular}{lcccccc} 
Brain volumes & Beta & $\boldsymbol{p}$ & R-square & Beta & $\boldsymbol{p}$ & R-square \\
\hline Whole & -.769 & $<.001$ & .525 & -.542 & $\mathbf{. 0 0 2}$ & .588 \\
hippocampus & -.483 & $\mathbf{. 0 1 4}$ & .359 & -.306 & .138 & .400 \\
Tail & -.651 & $\mathbf{. 0 0 1}$ & .442 & -.511 & $\mathbf{. 0 1 2}$ & .444 \\
Subiculum & -.684 & $<.001$ & .461 & -.526 & $\mathbf{. 0 0 7}$ & .495 \\
Presubiculum & -.645 & $\mathbf{. 0 0 1}$ & .422 & -.432 & $\mathbf{. 0 2 7}$ & .482 \\
Parasubiculum & -.190 & .353 & .269 & -.267 & .263 & .190 \\
Fissure & -.649 & $<.001$ & .488 & -.423 & $\mathbf{. 0 2 1}$ & .541 \\
CA1 & -.611 & $\mathbf{. 0 0 3}$ & .345 & -.470 & $\mathbf{. 0 2 8}$ & .371 \\
CA2-3 & -.670 & $\mathbf{. 0 0 1}$ & .351 & -.469 & $\mathbf{. 0 2 4}$ & .415 \\
CA4 & -.776 & $<.001$ & .502 & -.557 & $\mathbf{. 0 0 2}$ & .566 \\
ML & -.687 & $\mathbf{. 0 0 1}$ & .402 & -.475 & $\mathbf{. 0 1 7}$ & .469 \\
GCDG & -.667 & $<.001$ & .46 & -.441 & $\mathbf{. 0 1 8}$ & .528 \\
HATA & -.462 & $\mathbf{. 0 3 1}$ & .24 & -.335 & .148 & .247 \\
Fimbria &
\end{tabular}

Note: Statistics: Linear regression analyses of group affiliation (AN vs. HC) and HS with two different adjustments for brain size: eTIV (estimated total intracranial volume) and total brain volume without ventricles. Group variable was coded $\mathrm{AN}=0$ and $\mathrm{HC}=1$. For both sets of analyses, covariates were age, depression score (BDI-II), scanner site and drug use. Variables presented in bold are significant after FDR correction for multiple comparisons. HS = Hippocampal subfields. $\mathrm{CA}=$ Cornu Ammonis. GCDG = Granule cell layer of the dentate gyrus. HATA = Hippocampus-amygdala transition area. 
Table 5: The association between hippocampal subfields and clinical measures in AN

\begin{tabular}{lcccccccc}
\hline & \multicolumn{2}{c}{$\boldsymbol{B M I - S D S}$} & \multicolumn{2}{c}{$\boldsymbol{E D E} \boldsymbol{Q}$} & \multicolumn{2}{c}{ BDI-II } & \multicolumn{2}{c}{ STAI-Y1 } \\
Brain volumes & $\mathbf{B e t a}$ & $\boldsymbol{p}$ & Beta & $\boldsymbol{p}$ & Beta & $\boldsymbol{p}$ & Beta & $\boldsymbol{p}$ \\
\hline Total GM & .136 & .293 & .180 & .232 & .242 & .107 & .066 & .677 \\
& & & & & & & & \\
Whole & & & & & & & & \\
hippocampus & -.270 & .115 & .124 & .494 & .565 & $<.001$ & .567 & $\mathbf{. 0 0 1}$ \\
Tail & .137 & .489 & .025 & .906 & .346 & .084 & .334 & .105 \\
Subiculum & -.321 & .058 & .152 & .405 & .612 & $<.001$ & .619 & $<.001$ \\
Presubiculum & -.177 & .334 & .109 & .591 & .595 & $\mathbf{. 0 0 1}$ & .446 & $\mathbf{. 0 2 1}$ \\
Parasubiculum & .169 & .425 & .235 & .308 & .617 & $\mathbf{. 0 0 3}$ & .487 & $\mathbf{. 0 2 8}$ \\
CA1 & -.204 & .238 & .178 & .313 & .473 & $\mathbf{. 0 0 4}$ & .522 & $\mathbf{. 0 0 3}$ \\
CA2-3 & -.222 & .186 & .017 & .927 & .264 & .160 & .338 & .074 \\
CA4 & -.352 & .049 & .047 & .811 & .477 & $\mathbf{. 0 1 2}$ & .488 & $\mathbf{. 0 1 1}$ \\
ML & -.291 & .092 & .140 & .442 & .557 & $\mathbf{. 0 0 1}$ & .582 & $\mathbf{. 0 0 1}$ \\
GCDG & -.341 & .050 & .055 & .775 & .486 & $\mathbf{. 0 0 8}$ & .496 & $\mathbf{. 0 0 7}$ \\
HATA & -.140 & .436 & .094 & .629 & .521 & $\mathbf{. 0 0 4}$ & .452 & $\mathbf{. 0 1 6}$ \\
Fimbria & -.080 & .681 & .121 & .539 & .438 & .048 & .284 & .219 \\
\hline
\end{tabular}

Note: Statistics: Linear regression adjusting for age. site. drug use and eTIV. Variables presented in bold are significant at the 5\% level after FDR correction for multiple comparisons. BMI-SDS:

Standardized body mass index (BMI) values based on Norwegian norms for children. BDI-II: Becks depression inventory II. EDE-Q: Eating disorder examination questionnaire (global score). STAI: State Trait Anxiety Inventory form Y1 (State anxiety) and Y2 (Trait anxiety). CA = Cornu Ammonis. GCDG $=$ Granule cell layer of the dentate gyrus. HATA $=$ Hippocampus-amygdala transition area . 